\title{
Article \\ Eulerian Description of Wave-Induced Stokes Drift Effect on Tracer Transport
}

\author{
Sheng Yan ${ }^{1,2,3}$ (D) Zhili Zou ${ }^{3}$ and Zaijin You ${ }^{1,2, *(\mathbb{D})}$ \\ 1 College of Transport Engineering, Dalian Maritime University, Dalian 116026, China; \\ yansheng20210039@dlmu.edu.cn \\ 2 Centre for Ports and Maritime Safety, Dalian Maritime University, Dalian 116026, China \\ 3 State Key Laboratory of Coastal and Offshore Engineering, Dalian University of Technology, \\ Dalian 116024, China; zlzou@dlut.edu.cn \\ * Correspondence: b.you@dlmu.edu.cn
}

\section{check for}

updates

Citation: Yan, S.; Zou, Z.; You, Z Eulerian Description of

Wave-Induced Stokes Drift Effect on Tracer Transport. J. Mar. Sci. Eng. 2022, 10, 253. https://doi.org/ $10.3390 /$ jmse10020253

Received: 24 December 2021

Accepted: 7 February 2022

Published: 12 February 2022

Publisher's Note: MDPI stays neutral with regard to jurisdictional claims in published maps and institutional affiliations.

Copyright: (c) 2022 by the authors. Licensee MDPI, Basel, Switzerland. This article is an open access article distributed under the terms and conditions of the Creative Commons Attribution (CC BY) license (https:// creativecommons.org/licenses/by/ $4.0 /)$.

\begin{abstract}
The wave-induced Stokes drift plays a significant role on mass/tracer transport in the ocean and the evolution of coastal morphology. The tracer advection diffusion equation needs to be modified for Eulerian ocean models to properly account for the surface wave effects. The Eulerian description of Stokes drift effect on the tracer transport is derived in this study to show that this effect can be accounted for automatically in the wave-averaged advection-diffusion equation. The advection term in this equation is the wave-averaged concentration flux produced by the interaction between fluctuations of linear wave orbital velocity and tracer concentration, and the advection velocity is the same as the Stokes drift velocity. Thus, the effective dispersion of tracers by surface gravity waves is calculated due to the Stokes drift effect and the corresponding dispersion coefficient in the depth-integrated equation is then derived. The Eulerian description of Stokes drift effect of tracer concentration is illustrated by the direct numerical simulation of the advection-diffusion equation under simple linear waves. The equivalence between both the Eulerian and Lagrangian descriptions is also verified by particle tracking method. The theoretical analysis is found to agree well with the wave-induced dye drift velocity observed outside the surf zone in a longshore current experiment.
\end{abstract}

Keywords: Stokes drift velocity; mass transport; advection diffusion; tracer transport; Taylor dispersion; Eulerian velocity; Lagrangian velocity

\section{Introduction}

The Stokes drift velocity, which is the time mean velocity of a fluid particle in water waves [1,2], may play a significant role on the mass/tracer transport (e.g., larval, plankton, oil, plastic pollution, etc.) in the ocean and the evolution of coastal morphology. For instance, Monismith and Fong [3] showed through two field experiments that the onshore Stokes drift near the water surface could be stronger than measured Eulerian currents on the inner shelf, and it is necessary to include the Stokes drift to explain an observed shoreward migration of a dye plume trajectory. Although the Lagrangian Stokes drift cannot be detected directly by current measurements at a fixed position, it is suggested to be explicitly considered in the models that attempt to predict the transport of organisms and solutes in the nearshore environment as a surface wave effect. Rohrs et al. [4] presented that the Stokes drift exhibits a more constant direction in the long term than the Eulerian current, and the average distance to the coast of Northeast Arctic cod eggs and larvae decreases by $20 \%$ on average due to the wave effects. Reniers et al. [5] showed that when Stokes drift was considered in the model of the computed drifter trajectories, good agreement was found with the observed retention of surf zone material on a rip-channel beach. Without Stokes drift considered, however, the estimated results overpredicted the number of observed drifter exits by almost an order of magnitude during the drifter deployments. This emphasizes the importance of including Stokes drift when calculating 
the transport of floating materials on the ocean surface. Henderson et al. [6] showed that the Stokes drift could increase shoreward transport of suspended sediment and contribute to the shoreward bar migration. The Stokes drift is also a cross-shore exchange mechanism between the surf zone and the inner shore. Its importance relative to the transient rip currents was examined by using temperature and dye measurements at an approximately alongshore-uniform beach [7]. The dye release was simulated by the wave-resolving funwaveC model, but it does not include Stokes-drift driven exchange [8]. The tracer advection equation has been modified for Eulerian ocean models to account for Stokes drift effect by McWilliams et al. [9], but they did not consider the diffusion terms.

The variation of the Stokes drift velocity with water depth gives rise to the Taylor dispersive effect [10] in the longitudinal direction. Law [11] studied the dispersion of pollutants in the free surface waves. By assuming that the particles move passively with the water particles in the same phase and amplitude, the time-averaged velocity profile along the water depth was taken as the Stokes drift velocity profile. Taylor dispersion coefficient is obtained by using the formula derived by Van den Broeck [12]. A random walk method was also used to simulate the movement of pollutant particles in the surface waves. In the process of solving the particle position, the particle displacement at each time step is obtained by time integration of the instantaneous wave orbital velocities, so the effect of Stokes drift velocity is included. The results of the dispersion coefficients obtained by the above two methods are very close for the steady state, which indicates that the two methods are equivalent to describe the Stokes drift effect of pollutant diffusion under waves and the effects of wavy surface boundary can be ignored. For theoretical analysis, Shen and Zou [13] took the advection velocity in the vertical two-dimensional advective diffusion equation as the sum of the Stokes drift velocity and the linear wave orbital velocity, and derived the Taylor dispersion caused by the surface waves using a successive approximation method. The wave-averaged dispersion coefficient in their result is $35 \%$ larger than that of Law [11]. However, the sum of the Stokes drift velocity and the linear wave orbital velocity may be not properly used in the advection diffusion equation for the wave resolving concentration. Assuming the pollutant to be vertically well mixed, Winckler et al. [14] derived a two-dimensional depth-averaged advection diffusion equation for wave-averaged concentration in the shallow water of surf zone. Their theory shows that in addition to longshore current velocity induced by wave breakings, the advection velocities also include contributions from the depth-averaged Stokes drift velocities, which are due to the covariance of waves and fluctuations of the pollutant concentration. Within the vertically well-mixed surf zone, Stokes drift was found to be a negligible cross-shore dye dispersion mechanism relative to surf zone eddies [15]. However, outside the surf zone on the inner-shelf, vertically varying Stokes drift velocity could potentially be an important cross-shore dispersion mechanism.

In tracer transport models, the Stokes drift of gravity surface waves is usually considered using Lagrangian description [4,5]. However, the Eulerian concentration transport equation is also commonly used, so a Eulerian description for Stokes drift effect is necessary. This paper explores how to consider the effects of the Stokes drift in the Eulerian advective diffusion equation of tracer concentration and discusses the equivalence of the Eulerian description of the Stokes drift effect to the Lagrangian method. A multiple-scale analysis is used to derive the wave-averaged vertical two-dimensional and depth-averaged onedimensional advective diffusion equations of tracer transport in wave field. The Eulerian description of Stokes drift effect of tracer concentration is illustrated by numerically solving the advection diffusion equation in linear wave field directly. The theoretical results are also compared with the results from particle tracking method based on the Lagrangian description. A laboratory experiment is also presented to show that the dye drift induced by oblique incident waves was obvious outside the surf zone on a planar beach. 


\section{Eulerian Description of Stokes Drift Effect}

The generation mechanism of Stokes drift effect on the tracer transport in the wave field by the Eulerian description is given in this section. A multiple-scale analysis [16] is used for advection diffusion equation of the tracer concentration in the surface gravity wave field to obtain the transport equation of the wave-averaged concentration.

We assume that no chemical reaction is presented, and the tracer density is the same as that of the surrounding fluid or the sea water. The concentration of passive and conservative tracer, $c(x, z, t)$, in the gravity waves is assumed to be governed by the advection diffusion equation [17]

$$
\frac{\partial c}{\partial t}+u \frac{\partial c}{\partial x}+w \frac{\partial c}{\partial z}=\frac{\partial}{\partial x}\left(D_{x} \frac{\partial c}{\partial x}\right)+\frac{\partial}{\partial z}\left(D_{z} \frac{\partial c}{\partial z}\right),
$$

where $x$ and $z$ represent the horizontal and vertical coordinates, $u$ and $w$ represent the horizontal and vertical velocity components, $D_{x}$ and $D_{z}$ are the horizontal and vertical diffusivities assumed to be constant.

No-flux boundary conditions are applied to the impermeable bottom $z=-h$ and water surface $z=\eta$ :

$$
\begin{gathered}
\left(D_{x} \frac{\partial c}{\partial x}\right) \frac{\partial h}{\partial x}+\left(D_{\mathrm{z}} \frac{\partial c}{\partial z}\right)=0, \text { at the bottom } z=-h, \\
\left(D_{x} \frac{\partial c}{\partial x}\right) \frac{\partial \eta}{\partial x}-\left(D_{z} \frac{\partial c}{\partial z}\right)=0, \text { at the water surface } z=\eta(x, z, t) .
\end{gathered}
$$

According to the characteristics of waves and tracer transportation outside the surf zone, the normalization of following variables is introduced:

$$
\begin{aligned}
& x^{*}=k x, z^{*}=z / h, u^{*}=u T / A, w^{*}=w T / k h A, \\
& t^{*}=t / T, \eta^{*}=\eta / A, c^{*}=c / c_{0}, D^{*}=D / D_{v},
\end{aligned}
$$

where $A, k, T, h, c_{0}$, and $D_{v}$ are characteristic wave amplitude, wave number, wave period, water depth, tracer concentration, and vertical diffusivity, respectively. In terms of these dimensionless variables, Equation (1) can be rewritten non-dimensionally as:

$$
\frac{\partial c^{*}}{\partial t^{*}}+k A\left(u^{*} \frac{\partial c^{*}}{\partial x^{*}}+w^{*} \frac{\partial c^{*}}{\partial z^{*}}\right)=(k h)^{2} \frac{T D_{v}}{h^{2}} \frac{\partial}{\partial x^{*}}\left(D^{*} \frac{\partial c^{*}}{\partial x^{*}}\right)+\frac{T D_{v}}{h^{2}} \frac{\partial}{\partial z^{*}}\left(D^{*} \frac{\partial c^{*}}{\partial z^{*}}\right) .
$$

As the small-amplitude wave and the finite water depth outside the surf zone are considered, so $\varepsilon=k A$ and $k h=O(1)$ are assumed. Many field studies reported that the vertical diffusivity is small (e.g., $D_{v}=10^{-4} \sim 5 \times 10^{-3} \mathrm{~m}^{2} / \mathrm{s}$ [18]), so the ratio of wave period $T$ to the mixing time $h^{2} / D_{v}$ in a finite water depth(namely a time-scale for achieving a uniform condition between bottom and water surface) $T D_{v} / h^{2} \sim O\left(\varepsilon^{2}\right)$ is assumed. For example, considering the following characteristics of waves and vertical diffusivity: $h=3 \mathrm{~m}, H=0.6 \mathrm{~m}, T=5 \mathrm{~s}, D_{v}=0.005 \mathrm{~m}^{2} / \mathrm{s}$, then $k h=0.75 \sim O(1), T D_{v} / h^{2}=0.002778$, $\varepsilon^{2}=0.005715$, so we have $T D_{v} / h^{2} \sim O\left(\varepsilon^{2}\right)$.

Having identified the orders of these terms in Equation (5) and neglecting the higherorder terms, Equation (5) is then rewritten dimensionally, but in the simplified form of Equation (1), by introducing an ordering parameter $\varepsilon$,

$$
\frac{\partial c}{\partial t}+\varepsilon u \frac{\partial c}{\partial x}+\varepsilon w \frac{\partial c}{\partial z}=\varepsilon^{2} \frac{\partial}{\partial x}\left(D_{x} \frac{\partial c}{\partial x}\right)+\varepsilon^{2} \frac{\partial}{\partial z}\left(D_{z} \frac{\partial c}{\partial z}\right) .
$$

where the boundary conditions in Equations (2) and (3) are rescaled as:

$$
\begin{gathered}
\frac{\partial c}{\partial z}=0, z=-h, \\
\frac{\partial c}{\partial z}=\varepsilon \frac{\partial c}{\partial x} \frac{\partial \eta}{\partial x}-\varepsilon \frac{\partial^{2} c}{\partial z^{2}} \eta+o(\varepsilon), z=0 .
\end{gathered}
$$


The order contrast among the terms in Equation (6) indicates that there are two distinct timescales in the advective diffusion process of passive tracer. One is wave period $T$, which characterizes the fast oscillations of tracer caused by surface gravity waves, and the other is mixing time $h^{2} / D_{v}$ in the finite water depth, which characterizes the slow diffusion caused by the diffusivities. The ratio between the two timescales is $T D_{v} / h^{2} \sim O\left(\varepsilon^{2}\right)$. Because of the significant difference between the time scales of wave period and diffusion, we introduce multiple-scale coordinates for time: $t$ for the wave motion and $\tau=\varepsilon^{2} t$ for the effects of diffusion. Thus, the tracer concentration $c(x, z, t, \tau)$ depends on $(t, \tau)$ as independent variables. The original time derivative becomes, according to the chain rule, $\partial c / \partial t \rightarrow \partial c / \partial t+\varepsilon^{2} \partial c / \partial \tau$. The wave velocity, wave surface and tracer concentration are expanded as follows:

$$
\begin{gathered}
u=u^{(1)}+\varepsilon u^{(2)}+O\left(\varepsilon^{2}\right), \\
w=w^{(1)}+\varepsilon w^{(2)}+O\left(\varepsilon^{2}\right), \\
\eta=\eta^{(1)}+\varepsilon \eta^{(2)}+O\left(\varepsilon^{2}\right), \\
c=c^{(0)}+\varepsilon c^{(1)}+\varepsilon^{2} c^{(2)}+O\left(\varepsilon^{3}\right),
\end{gathered}
$$

where $u^{(n)}, w^{(n)}$ and $\eta^{(n)}$ are $n$th harmonics of wave horizontal and vertical orbital velocities and surface wave profile, and $c^{(n)}=c^{(n)}(x, z, t, \tau)$. Stokes wave theory [19] is used, and we have

$$
\begin{gathered}
u^{(1)}=\frac{A \omega}{\sinh (k h)} \cosh k(h+z) \cos (k x-\omega t), \\
w^{(1)}=\frac{A \omega}{\sinh (k h)} \sinh k(h+z) \sin (k x-\omega t), \\
\eta^{(1)}=A \cos (k x-\omega t),
\end{gathered}
$$

and $u^{(2)}, w^{(2)}$ and $\eta^{(2)}$ are second harmonics. Equating the coefficient of each power of $\varepsilon$ to zero leads to a sequence of perturbation equations. At the leading order $c^{(0)}(x, z, \tau)$ is expected to represent the period average and depends only on $\tau$. The transport equation at $O(1)$ is trivially satisfied by the period-averaged $c^{(0)}(x, z, \tau)$ and $\partial c^{(0)} / \partial z=0$ at $z=0$ and $z=-h$. At $O(\varepsilon), c^{(1)}(x, z, t, \tau)$ represents the perturbation due to the oscillating velocity field and satisfies

$$
\frac{\partial c^{(1)}}{\partial t}=-u^{(1)} \frac{\partial c^{(0)}}{\partial x}-w^{(1)} \frac{\partial c^{(0)}}{\partial z}
$$

and the boundary conditions:

$$
\frac{\partial c^{(1)}}{\partial z}=0, z=-h
$$

and

$$
\frac{\partial c^{(1)}}{\partial z}=\frac{\partial c^{(0)}}{\partial x} \frac{\partial \eta^{(1)}}{\partial x}-\frac{\partial^{2} c^{(0)}}{\partial z^{2}} \eta^{(1)}, z=0
$$

At $O\left(\varepsilon^{2}\right), c^{(2)}$ satisfies

$$
\frac{\partial c^{(2)}}{\partial t}-\frac{\partial}{\partial x}\left(D_{x} \frac{\partial c^{(0)}}{\partial x}\right)-\frac{\partial}{\partial z}\left(D_{z} \frac{\partial c^{(0)}}{\partial z}\right)=-\frac{\partial c^{(0)}}{\partial \tau}-u^{(1)} \frac{\partial c^{(1)}}{\partial x}-w^{(1)} \frac{\partial c^{(1)}}{\partial z}-u^{(2)} \frac{\partial c^{(0)}}{\partial x_{i}}-w^{(2)} \frac{\partial c^{(0)}}{\partial z}
$$

and the boundary conditions

$$
\frac{\partial c^{(2)}}{\partial z}=0, z=-h
$$

and

$$
\frac{\partial c^{(2)}}{\partial z}=\varepsilon \frac{\partial c^{(0)}}{\partial x} \frac{\partial \eta^{(2)}}{\partial x}-\varepsilon \frac{\partial^{2} c^{(0)}}{\partial z^{2}} \eta^{(2)}+\varepsilon \frac{\partial c^{(1)}}{\partial x} \frac{\partial \eta^{(1)}}{\partial x}-\varepsilon \frac{\partial^{2} c^{(1)}}{\partial z^{2}} \eta^{(1)}, z=0
$$

Substituting Equations (13), (14) into Equation (16), we get 


$$
c^{(1)}\left(x_{i}, z, t, \tau\right)=\frac{A}{\sinh (k h)}\left[\cosh k(h+z) \frac{\partial c^{(0)}}{\partial x} \sin (k x-\omega t)-\sinh k(h+z) \frac{\partial c^{(0)}}{\partial z} \cos (k x-\omega t)\right] .
$$

Equation (19) has a solution $c^{(2)}$ that is periodic in $x$ and $t$ if and only if the following solvability condition holds:

$$
\frac{\partial c^{(0)}}{\partial \tau}-\frac{\partial}{\partial x}\left(D_{x} \frac{\partial c^{(0)}}{\partial x}\right)-\frac{\partial}{\partial z}\left(D_{z} \frac{\partial c^{(0)}}{\partial z}\right)=\overline{-u^{(1)} \frac{\partial c^{(1)}}{\partial x}}-\overline{w^{(1)} \frac{\partial c^{(1)}}{\partial z}},
$$

where denoting the wave-average by overline, $\bar{f}=\int_{t}^{t+T} f \mathrm{~d} t / T$.

Substituting Equation (22) into Equation (19), we get

$$
\frac{\partial c^{(0)}(x, z, \tau)}{\partial \tau}+u_{c} \frac{\partial c^{(0)}(x, z, \tau)}{\partial x}=\frac{\partial}{\partial x}\left(D_{x} \frac{\partial c^{(0)}(x, z, \tau)}{\partial x}\right)+\frac{\partial}{\partial z}\left(D_{z} \frac{\partial c^{(0)}(x, z, \tau)}{\partial z}\right) .
$$

This equation is a transport equation for wave-averaged concentration, $c^{(0)}$, with the advection velocity

$$
u_{c}=\frac{A^{2} \omega k \cosh 2 k(z+h)}{2 \sinh ^{2} k h} .
$$

Notice that the advection term, $u_{c} \partial c(0) / \partial x$, is the phase-averaged concentration flux produced by interaction between fluctuations of linear wave orbital velocities and tracer concentration

$$
u_{c} \frac{\partial c^{(0)}}{\partial x}=\overline{\left(u^{(1)} \frac{\partial c^{(1)}}{\partial x}+w^{(1)} \frac{\partial c^{(1)}}{\partial z}\right)}
$$

and the advection velocity is identical in form to the Stokes drift velocity with the definition given by Longuet-Higgins [2]

$$
u_{s}=\overline{\int^{t} u^{(1)} \mathrm{d} t \frac{\partial u^{(1)}}{\partial x}+\int^{t} w^{(1)} \mathrm{d} t \frac{\partial u^{(1)}}{\partial x}}=\frac{A^{2} \omega k \cosh 2 k(z+h)}{2 \sinh ^{2} k h} .
$$

This means that the wave-averaged transport in wave velocity field can be expressed by the transport by Stokes drift. The former Equation (25) is a Eulerian description whereas the latter Equation (27) is a Lagrangian description, which means the two are equivalent for the case of considering the wave-averaged tracer transport problem.

The variation of the advection velocity with water depth gives rise to the Taylor dispersive effect [10] in the longitudinal direction, and a dispersion effect will also be generated in the depth-averaged equation

$$
\frac{\partial c_{a}^{(0)}(x, \tau)}{\partial \tau}+U \frac{\partial c_{a}^{(0)}(x, \tau)}{\partial x}=\left(D_{x}+K\right) \frac{\partial^{2} c_{a}^{(0)}(x, \tau)}{\partial x^{2}}
$$

where $c_{a}^{(0)}$ is the depth-averaged tracer concentration, $U$ is the depth-averaged velocity, $K$ is the Taylor dispersion coefficient when the dispersing cloud becomes normally distributed approximately. Their expressions are:

$$
\begin{gathered}
c_{a}^{(0)}(x, \tau)=\frac{1}{h} \int_{-h}^{0} c^{(0)}(x, z, \tau) \mathrm{d} z, \\
U=\frac{1}{h} \int_{-h}^{0} u_{c} \mathrm{~d} z=\frac{A^{2} \omega}{2 h \tanh ^{2} h^{\prime}}
\end{gathered}
$$




$$
\begin{aligned}
& K=\frac{-1}{h \partial c_{a}^{(0)} / \partial x} \int_{-h}^{0} \hat{u}_{c} \hat{c}^{(0)} \mathrm{d} z \\
& =\frac{-1}{h D_{z}} \int_{-h}^{0} \hat{u}_{c} \int_{-h}^{z} \int_{-h}^{z} \hat{u}_{c} \mathrm{~d} z \mathrm{~d} z \mathrm{~d} z \\
& =\frac{H^{4}}{D_{z} T^{2}} \frac{\pi^{2}}{16 \tanh ^{2} k h}\left(\frac{\sinh 4 k h-4 k h}{4 k h(\cosh 4 k h-1)}+\frac{1}{2(k h)^{2}}-\frac{\cosh 2 k h}{k h \sinh 2 k h}+\frac{1}{3}\right)
\end{aligned}
$$

where $\hat{c}^{(0)}$ and $\hat{u}_{c}$ are deviations of $c^{(0)}$ and $u_{c}$ from their depth-averaged values. The expression for $K$ is the same as that was derived analytically by Law [11] using a different method of Van den Broeck [12].

Chatwin [20] has also shown that the dispersing cloud will become normally distributed approximately a time $h^{2} / D_{z}$ after its injection. If the concentration at time $h^{2} / D_{z}$

$$
c_{a}^{(0)}\left(x, h^{2} / D_{\mathrm{z}}\right)=\frac{M}{\sqrt{2 \pi} \sigma} \exp \left(-\frac{x^{2}}{2 \sigma^{2}}\right)
$$

is the initial condition for Equation (28) ( $M$ represents the total amount of the diffusing substance and $\sigma^{2}$ is the variance), the solution of Equation (28) can be expressed as

$$
c_{a}^{(0)}(x, \tau)=\frac{M}{\sqrt{2 \pi\left(\sigma^{2}+2 K t\right)}} \exp \left(-\frac{(x-U t)^{2}}{2\left(\sigma^{2}+2 K t\right)}\right) .
$$

At this stage, the deviation of concentration can be given by $\hat{c}^{(0)}$, then

$$
\begin{aligned}
& c^{(0)}(x, z, \tau)=c_{a}^{(0)}(x, \tau)+\hat{c}^{(0)} \\
& =c_{a}^{(0)}(x, \tau)+\frac{1}{D_{\mathrm{z}}} \frac{\partial c_{a}^{(0)}}{\partial x} \frac{H^{2} k \pi}{4 T}\left(\frac{1}{4 k^{2}} \frac{\cosh 2 k(z+h)}{\sinh ^{2} k h}-\frac{z^{2}+2 h z}{2 k h \tanh k h}\right. \\
& \left.-\frac{1}{4 k^{2} \sinh ^{2} k h}-\frac{h}{2 k \tanh k h}\right)+\hat{c}^{(0)}(x,-h, \tau) \\
& =c_{a}^{(0)}(x, \tau)+\frac{1}{D_{\mathrm{z}}} \frac{\partial c_{a}^{(0)}}{\partial x} \frac{H^{2} k \pi}{4 T}\left(\frac{1}{4 k^{2}} \frac{\cosh 2 k(z+h)}{\sinh ^{2} k h}-\frac{z^{2}+2 h z}{2 k h \tanh k h}\right. \\
& \left.-\frac{\sinh 2 k h}{8 k^{3} h \sinh ^{2} k h}-\frac{h}{12 k \tanh k h}\right),
\end{aligned}
$$

where $\hat{c}^{(0)}(x,-h, \tau)$ is determined by $\int_{-h}^{0} \hat{c}^{(0)}(x, z, \tau) \mathrm{d} z=0$. At this stage, a theoretical solution to Equation (1) can be derived as:

$$
\begin{aligned}
& c(x, z, t, \tau)=c^{(0)}(x, z, \tau)+c^{(1)}(x, z, t, \tau) \\
& =c^{(0)}(x, z, \tau)+\frac{A}{\sinh (k h)}\left[\cosh k(h+z) \frac{\partial c^{(0)}}{\partial x} \sin (k x-\omega t)-\sinh k(h+z) \frac{\partial c^{(0)}}{\partial z} \cos (k x-\omega t)\right] .
\end{aligned}
$$

The following two sections will give the results of the direct numerical solution of the advection diffusion Equation (1) and the Lagrangian description results of the particle tracking method, and further explain the equivalence of above Eulerian description of the Stokes drift effect to Lagrangian description.

\section{Numerical Solution of the Advection Diffusion Equation}

In the previous Section 2, the advection term (26) in the advection diffusion equation of the period-averaged concentration comes from the interaction of the first-order wave orbital velocities and corresponding concentration fluctuation. In order to visualize the physical process described by the Eulerian description, the concentration is directly simulated by numerically solving Equation (1) in the first-order wave field given by Equations (13)-(15).

In order to consider the variation of the calculation domain caused by the wave free surface, the calculation domain is transformed into a fixed calculation domain through the following $\sigma$ coordinate transformation:

$$
\xi=x, \sigma=\frac{z+h}{h+\eta(x, t)}, t^{\prime}=t .
$$


By applying the chain rule to Equation (1), the advection diffusion equation in $\sigma$ coordinates can be expressed as:

$$
\begin{aligned}
& \frac{\partial c}{\partial t^{\prime}}+u \frac{\partial c}{\partial \xi^{2}}+\left(\frac{w}{h+\eta}-\frac{\sigma}{h+\eta} \frac{\partial \eta}{\partial t^{\prime}}-u \frac{\sigma}{h+\eta} \frac{\partial \eta}{\partial \xi}\right) \frac{\partial c}{\partial \sigma}+\left(D_{x} \frac{\partial^{2} \eta}{\partial \xi^{2}} \frac{\sigma}{h+\eta}-2\left(\frac{\partial \eta}{\partial \xi}\right)^{2} \frac{\sigma}{(h+\eta)^{2}}\right) \frac{\partial c}{\partial \sigma}= \\
& D_{x} \frac{\partial^{2} c}{\partial \xi^{2}}-2 D_{x} \frac{\sigma}{h+\eta} \frac{\partial \eta}{\partial \xi} \frac{\partial^{2} c}{\partial \xi^{2} \partial \sigma}+D_{x}\left(\frac{\sigma}{\eta+h} \frac{\partial \eta}{\partial \xi}\right)^{2} \frac{\partial^{2} c}{\partial \sigma^{2}}+D_{z} \frac{1}{(h+\eta)^{2}} \frac{\partial^{2} c}{\partial \sigma^{2}} .
\end{aligned}
$$

The up and down boundary condition can be expressed as:

$$
\frac{\partial c}{\partial \sigma}=0, \text { at } \sigma=0 \text { and } \sigma=1,
$$

and left and right boundary conditions are:

$$
c( \pm \infty, \sigma, t)=0 .
$$

The initial condition of a line source can be expressed by:

$$
c(\xi, \sigma, 0)=M \delta(\xi-20) / h,
$$

where $M$ represents the total amount of the diffusing substance, $\delta$ is a Dirac delta function, and the initial line source is at $x=20 \mathrm{~m}$.

The finite difference method is used to discrete the terms of Equation (37). ADI (alternating direction implicit) scheme is used to solve the equation, and the numerical scheme has been validated for a steady flow. In this calculation, water depth $h=3 \mathrm{~m}$, wave height $H=0.6 \mathrm{~m}$, wave period $T=5 \mathrm{~s}$, and $D_{x}=D_{z}=0.005 \mathrm{~m}^{2} / \mathrm{s}$ are used. The length of model domain is $80 \mathrm{~m}$. The grid spacing along the depth is $0.1 \mathrm{~m}$, and the grid spacing along the direction of wave propagation is $0.1 \mathrm{~m}$. The time step is $0.01 \mathrm{~s}$.

Figure 1 shows the concentration distribution in the flow field at time $t=800 \mathrm{~s}$ and the horizontal displacement of the concentration center caused by this Stokes drift effect. It can be seen that while the concentration diffuses under the action of waves, there is also a drift along the wave propagation direction. The drift velocity is the same as the Stokes drift velocity in Equation (27). This is illustrated here by estimating the depth-averaged velocity of this drift

$$
U=\frac{\int_{-\infty}^{+\infty} x C(x, t) \mathrm{d} x}{\Delta t \int_{-\infty}^{+\infty} C(x, t) \mathrm{d} x}=\frac{\sum_{i=1}^{801} i \Delta \xi \sum_{j=1}^{11} c(i, j)}{\Delta t \sum_{j=1}^{11} c(i, j)} \approx 0.03 \mathrm{~m} / \mathrm{s},
$$

where $C$ is the depth-averaged concentration, $c(i, j)$ is the concentration at the grid point $(i, j)$, and $\Delta \xi$ the horizontal grid spacing. The tracer concentration drift velocity obtained from Equation (41) is similar to the depth-averaged drift velocity of $0.03 \mathrm{~m} / \mathrm{s}$ obtained by Equation (30) and that by the particle tracking method under the same conditions.

The wave velocity field in the calculation is taken as the first-order wave orbital velocity components of Equations (13) and (14), but the second-order Stokes drift velocity is not considered. The numerical simulation has indicated that although the Stokes drift velocity is not added, the drift effect can be formed by the interaction of the first-order wave orbital velocity of the periodic wave and the concentration component caused by the periodic waves (i.e., caused by the convection term $u \partial c / \partial x+w \partial c / \partial z$ in Equation (1)). 
The initial position of line source

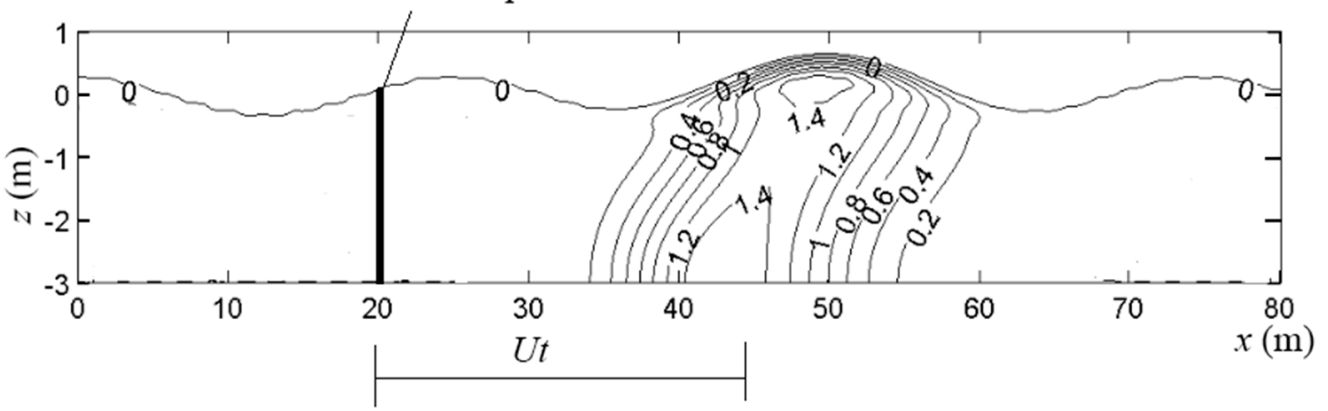

Figure 1. The tracer concentration evolution in a wave field after $800 \mathrm{~s}$ under the conditions of water depth $h=3 \mathrm{~m}$, wave height $H=0.6 \mathrm{~m}$, and wave period $T=5 \mathrm{~s}$.

\section{Modeling Tracer Transport by Particle Tracking Method}

The principle of particle tracking method is to calculate the trajectories of particles in the flow field and can be used to determine the concentration distribution. In this Lagrangian method, the Stokes drift is reflected by the wave-averaged particle displacement. The particle tracking method [21] uses the governing equation:

$$
\mathrm{d} \vec{Y}(t)=\left(\begin{array}{c}
u(\vec{Y}, t) \\
w(\vec{Y}, t)
\end{array}\right) \mathrm{d} t+\left[\begin{array}{cc}
\sqrt{2 D_{x}} & 0 \\
0 & \sqrt{2 D_{z}}
\end{array}\right]\left(\begin{array}{c}
A_{1} \\
A_{2}
\end{array}\right) \sqrt{\mathrm{d} t},
$$

where $\vec{Y}(t)=(x(t), z(t))$ are the coordinate of particle positions, $u(\vec{Y}, t)$ and $w(\vec{Y}, t)$ are the first-order wave orbital velocities, $A_{1}$ and $A_{2}$ are independent random numbers from the standard normal distribution with zero mean and unit variance, and $D_{x}$ and $D_{z}$ are the horizontal and vertical diffusion coefficients.

An example of simulating tracer transport in a surface wave field by particle tracking method is given here to show how the Stokes drift phenomenon is realized in the Lagrangian description. The calculation conditions of water depth, wave conditions and diffusion coefficients are taken as the same as those in Section 3. There are 6000 particles released in the wave field. The initial horizontal coordinates of the particles are located at $x=0$, and the random vertical coordinates are uniformly distributed along the water depth. The time step is $0.25 \mathrm{~s}$, and the spatial positions of the particles at any time can be calculated according to Equation (42). The fourth order Runge-Kutta method is used to solve Equation (42), and the second term is processed using the random walk method. The numerical scheme is validated by theoretical results of Taylor Dispersion in steady channel flow. If the particles move beyond the free surface boundary and the bottom boundary due to random walk, they are mirror reflected into the water domain. Figure 2 shows the spatial distribution of particles and concentration distribution in the linear wave field at $t=800 \mathrm{~s}$ corresponding to Figure 1. The horizontal center position of the particle distribution $\bar{x}$ can be obtained from the spatial position distribution of the particles, and the average speed $U$ of the overall particle drift can be obtained by dividing $\bar{x}$ by the corresponding time interval $t$. Figure 3 shows the value given by the Eulerian analytical solution of Equation (30) and that obtained by numerical simulation of $U$. The two values tend to be the same, which indicates that there is an overall drift with the velocity of Equation (30) as the advection velocity during the particle diffusion process in the wave field. 

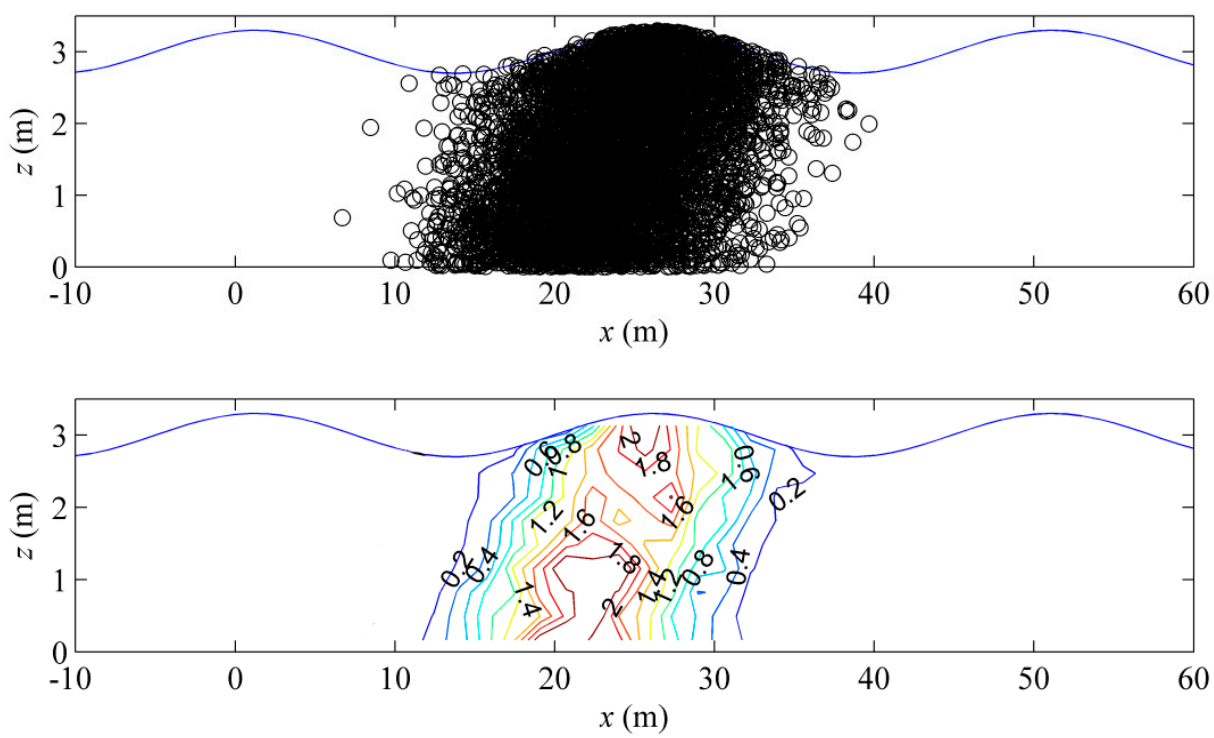

Figure 2. Simulation of particle drift in the linear wave field: particle distribution at $t=800 \mathrm{~s}$ (upper), and concentration distribution at $t=800 \mathrm{~s}$ (lower).

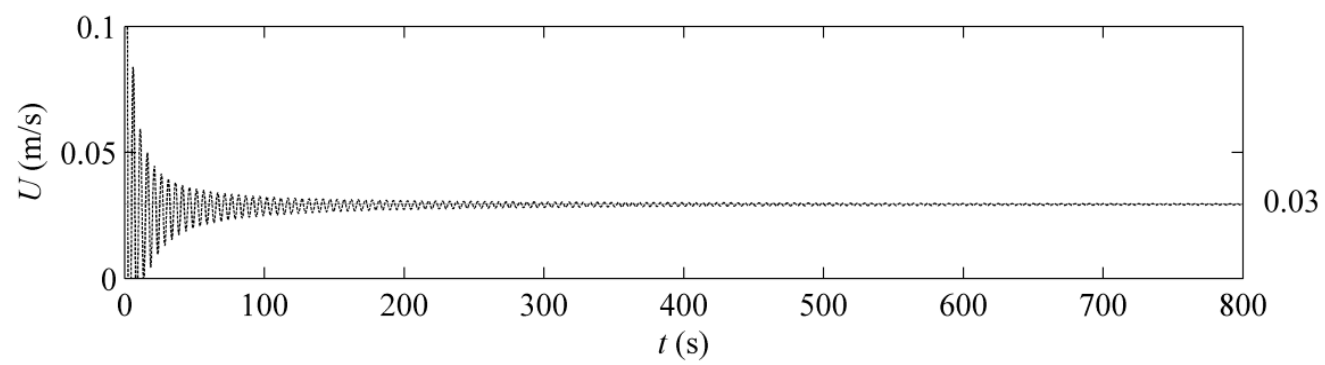

Figure 3. Stokes drift velocity obtained from simulation (dotted line) and analytical solution (Equation (30)) (thin solid line).

The reason for the above consistency is that the overall drift velocity $U$ of the particles is determined by the first term at the right end in Equation (42), while the second term does not affect the averaged motion in a statistical sense. Therefore, the Stokes drift velocity can be obtained only by the time integration of the first term (by tracking particles at different water depths). Figure 4 shows the distribution of the Stokes drift velocity along the water depth obtained by this Lagrangian method, and consistent with the analytical result from Equation (27).

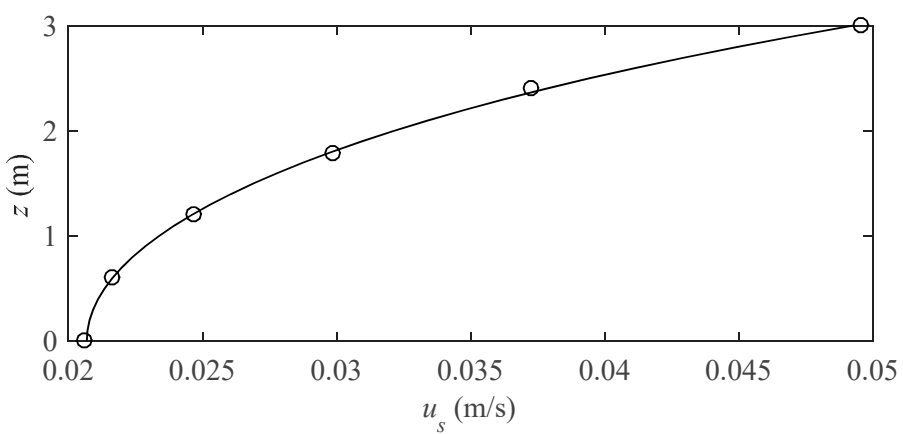

Figure 4. Simulated Stokes drift velocities (open circles) of the particles and analytical solution (Equation (27)) (thin solid line). 
From the above results, the dispersion coefficient in the depth-averaged Equation (28) can also be determined.

$$
K=\frac{\frac{1}{N} \sum_{i=1}^{N}\left(x_{i}-\bar{x}\right)^{2}}{2 t}-D_{x},
$$

where $\bar{x}$ is the horizontal center position of the particles, and $N$ is the number of simulated particles. The condition for the above formula to be applicable with reasonable accuracy is $t \geq 0.4 h^{2} / D_{z}$ [20], so the value calculated in the initial stage is not considered. Figure 5 shows the temporal variation of $K$ calculated by the above formula, which approaches a stable constant (about $K=0.0108 \mathrm{~m}^{2} / \mathrm{s}$ ) after the initial growth stage. The $K$ value is found to be very close to $K=0.0115 \mathrm{~m}^{2} / \mathrm{s}$, calculated from Equation (31). In this way, the dispersion effect expressed by Equation (31) caused by the linear surface wave field is illustrated well in Figures 2 and 5.

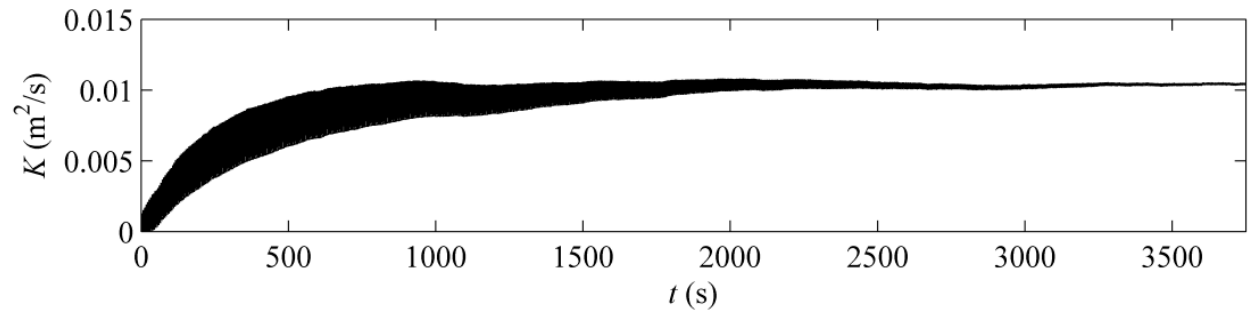

Figure 5. Temporal variation of dispersion coefficient $K$ under simple linear waves.

Figure 6 shows the spatial distribution of particles at the time $t=1800 \mathrm{~s}$ (upper) and the horizontal distribution of the number of particles (lower) and the corresponding theoretical normal distribution of probability density function. The results show that the particles can eventually be normally distributed along the horizontal direction when $t \geq h^{2} / D_{z}=1800 \mathrm{~s}$. This validates the assumption of Equation (32) under the effect of surface waves.
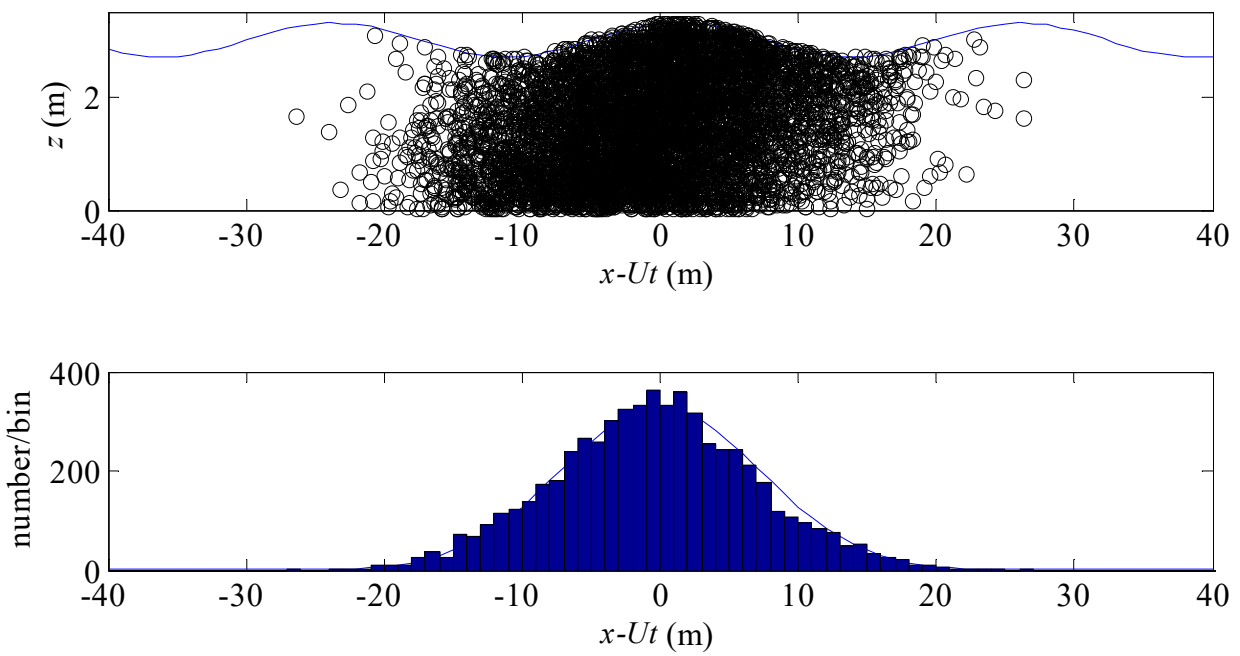

Figure 6. The spatial distribution of particles at the time $t=1800 \mathrm{~s}$ (upper) and the horizontal distribution of the number of particles with the corresponding theoretical normal distribution of probability density function (lower).

\section{Experimental Results and Discussion}

A laboratory experiment was conducted to study the effects of wave-induced currents on the dye transport outside and inside the surf zone [22]. The experiment was carried out in the wave basin of the State Key Laboratory of Coastal and Offshore Engineering. Figure 7 shows the plain view of the experimental setup. The wave basin is $55 \mathrm{~m}$ long, $34 \mathrm{~m}$ 
wide, and $0.8 \mathrm{~m}$ deep. At one end of the basin was a piston-type wave maker, and the other end was the wave absorber. A concrete beach model was built with a $30^{\circ}$ angle to the wave maker. The beach slope was 1:100, and the water depth at horizontal bottom was $0.18 \mathrm{~m}$. The incident wave height used in this paper was $0.05 \mathrm{~m}$, and the wave period was $1.5 \mathrm{~s}$. The regular waves were propagating at an angle of $30^{\circ}$ to the shore. The three measuring carriages on which wave gauges (at the sections $y=4 \mathrm{~m}, 8 \mathrm{~m}, 12 \mathrm{~m}$ ) and velocity meters (at the sections $y=8 \mathrm{~m}, 12 \mathrm{~m}$ ) were mounted were perpendicular to the shoreline.

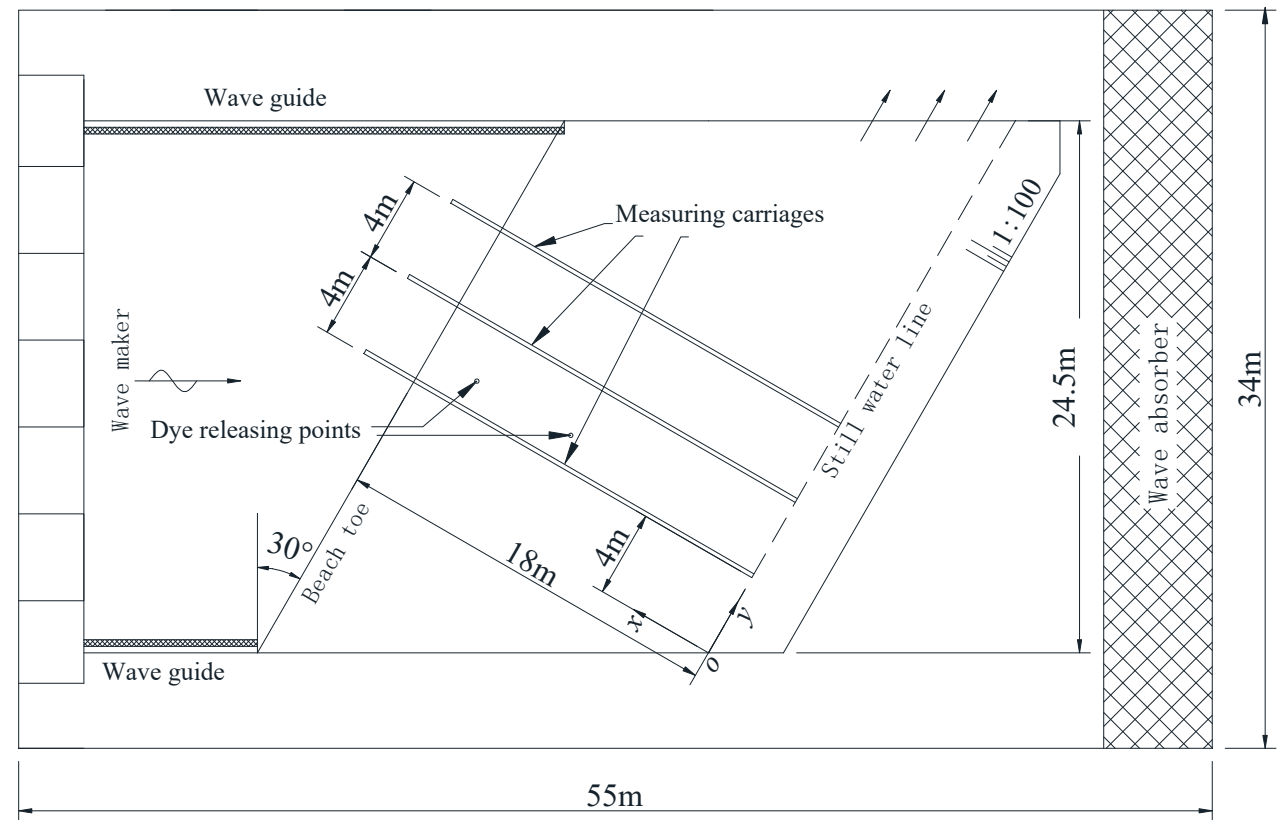

Figure 7. Plain view of the experimental setup.

The positions of the dye releasing points are at $x=10.5 \mathrm{~m}$ and $x=15.5 \mathrm{~m}$ which are outside the surf zone, and the wave breaking point is $9 \mathrm{~m}$ from the shoreline (see Figure $8 \mathrm{a}$ ). The dye was continuously released, and the dye-patch image was recorded by a chargecoupled device (CCD) at a height of about $8 \mathrm{~m}$ above the water surface. Figure 9 shows a series of CCD photos that was taken every $15 \mathrm{~s}$. These photos show that the dye exhibits a shoreward drift under the unbroken waves. As the mean Eulerian velocity outside the surf zone is nearly zero (see Figure 8 b), the drifting distance can be calculated by the Stokes drift velocity. The time interval between the fourth photo Figure $9 \mathrm{~d}$ and the first photo Figure $9 \mathrm{a}$ is $\Delta t=45 \mathrm{~s}$, and the drift velocity at the corresponding water surface $\left[u_{c}\right]_{z=0} \approx 0.048 \mathrm{~m} / \mathrm{s}$. It is calculated according to Equation (25) by taking the wave height at the midpoint of the dye trajectory line as $0.055 \mathrm{~m}$ and water depth of $h=0.1 \mathrm{~m}$. As the depth variation of Stokes effect is obvious outside the surf zone, the drift velocity at the corresponding water surface is used to calculate the horizontal length of the dye plume. The dye drifting distance from the two figures is $\Delta x=\int_{0}^{45} u_{c} \mathrm{~d} t \approx 2.36 \mathrm{~m}$. This distance is about 1.7 times the local wavelength of $1.44 \mathrm{~m}$. This result can be also seen from Figure 9, because the diagonal bright lines in the figure are the wave crest lines, and the dye movement distance from the first photo to the fourth photo is two times the distance between two wave crest lines (wavelength). This demonstrates that the dye drift outside the surf zone is mainly controlled by the Stokes drift effect. However, the dye is not only driven by the Stokes drift but also by the longshore current (the direction is parallel to the coast, see Figure $8 \mathrm{c}$ ) induced by the wave breaking after entering the surf zone. The dye trajectory tends to be parallel to the coast, which is shown in the last two photos in Figure 9e,f. 

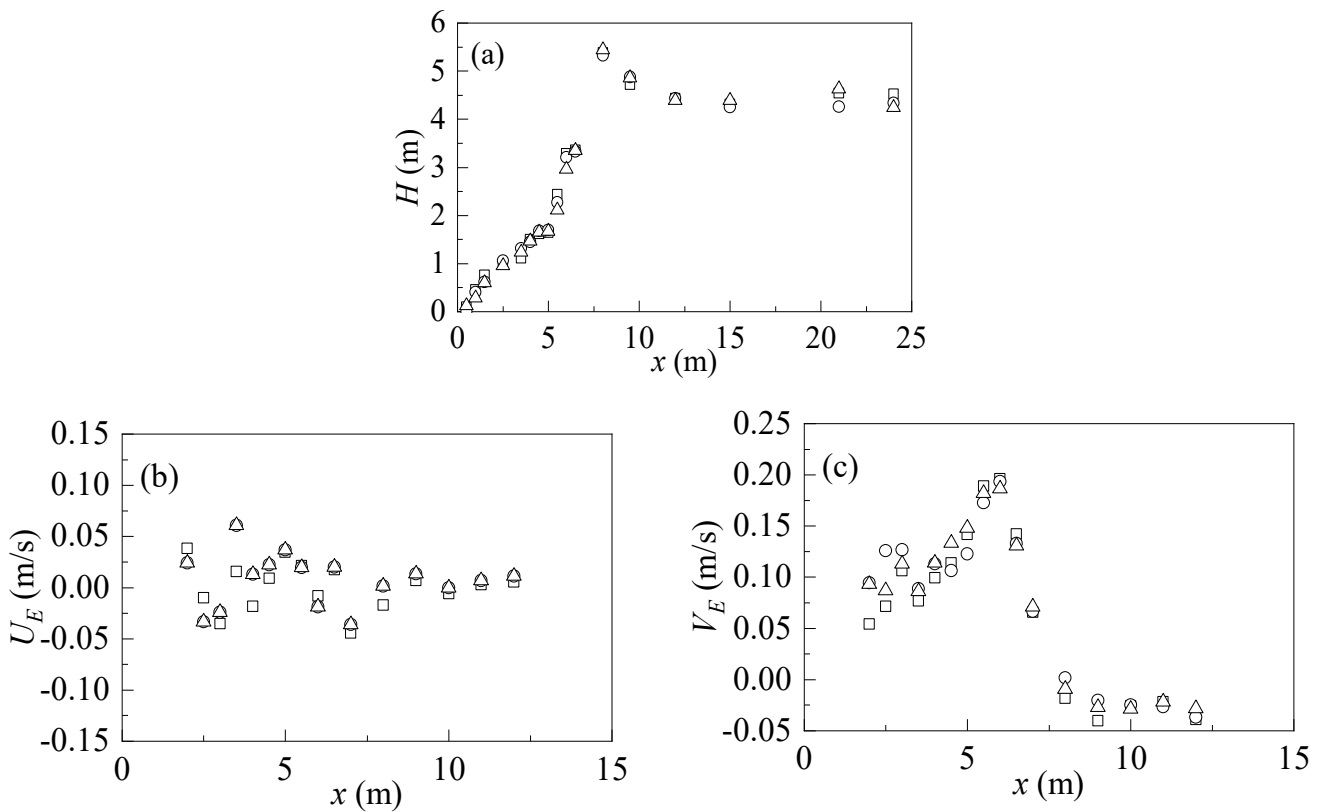

Figure 8. Measured cross-shore profiles of wave height $H(\mathbf{a})$, cross-shore current $U_{E}(\mathbf{b})$ and longshore current $V_{E}$ (c) at the section of $y=8 \mathrm{~m}$. The three type symbols represent the results from three repeated tests.
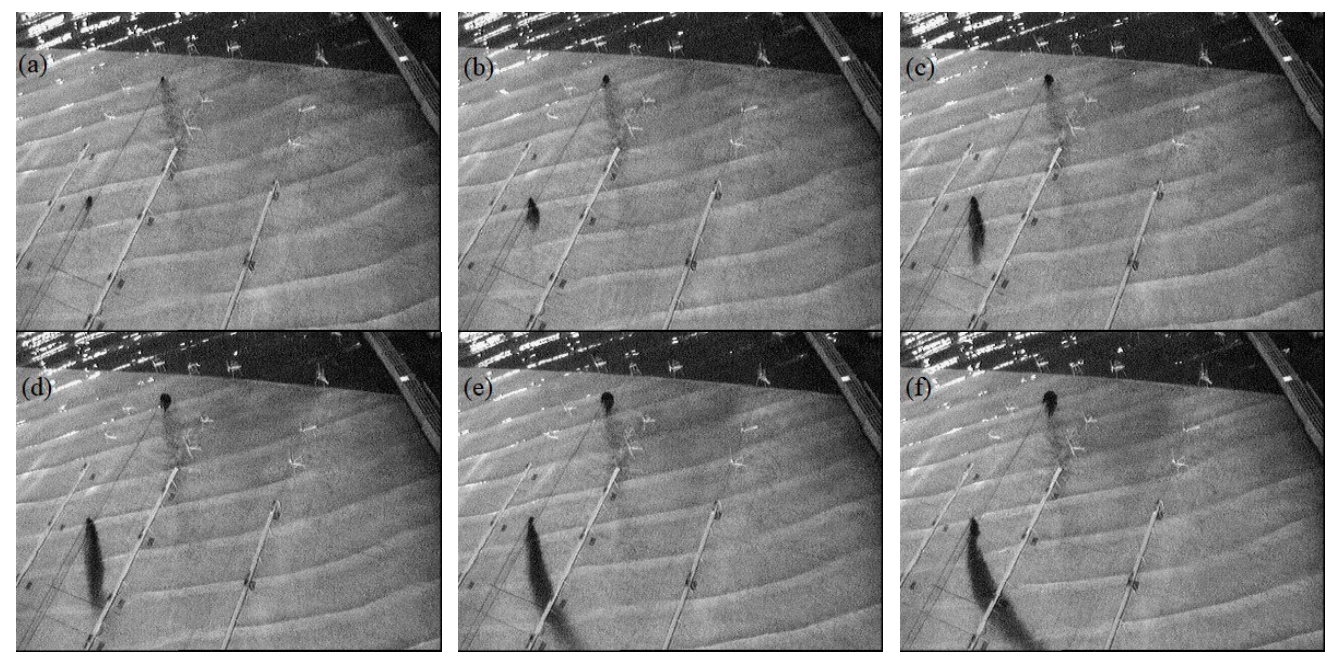

Figure 9. Trajectory of dye movement. The time interval between the adjacent photos is $15 \mathrm{~s}$. The three measuring carriages are perpendicular to the shoreline. (a) $0 \mathrm{~s}$, (b) $15 \mathrm{~s}$, (c) $30 \mathrm{~s}$, (d) $45 \mathrm{~s}$ (e) $60 \mathrm{~s}$, (f) $75 \mathrm{~s}$.

Long-term inner shelf observations [23] show that the offshore Eulerian flow can be roughly equal in magnitude but opposite in direction to the onshore Stokes drift at all depths, resulting in no net exchange. However, in the present experiment the effect of Stokes drift on dye transport is evident outside the surf zone when the incident waves are oblique to the coast, as the longshore current will take away the mass transport due to the Stokes drift. The measured mean Eulerian cross-shore velocity is smaller than the Stokes drift velocity outside the surf zone on inner shelf. This experiment is different from those experiments conducted in the wave flume [24,25] or basin [26] where incident waves are normal to the coast and the undertow or rip current velocity may decelerate the shoreward-moving tracers just outside the surf zone. Therefore, the above Stokes drift effect should be accounted for in the wave-averaged concentration flux of tracer along the wave direction besides the wave-averaged mean Eulerian velocity. This is also justified 
by the field observations given by Monismith [3] for the potential transport of solutes and organisms by surface waves.

\section{Conclusions}

This study quantitatively investigates the Eulerian description of the Stokes drift effect in the advection diffusion of tracer concentration under surface water waves. The Stokes drift effect is produced by the interaction between the periodic wave velocities and the periodic concentration variation and can be naturally included when the wave field is taken as the first-order wave orbital velocity. This effect appears explicitly in the wave-averaged advection diffusion equation of concentration as the advection velocity is just the Stokes drift velocity. This velocity shows that the concentration drift in the direction of wave propagation in addition to the periodic fluctuations induced by the surface waves. This process of tracer advective diffusion is verified by numerical calculation of the Eulerian vertical two-dimensional advective diffusion equation and the Lagrangian particle tracking method in this research. The Taylor dispersion coefficient produced by the Stokes drift effect in the depth-averaged equation is also obtained from the above theoretical analysis and is also consistent with the results obtained in the particle tracking method and previous studies. The Stokes drift effect by surface water waves outside the surf zone is shown to be significant by the photos of shoreward drifting of dye in the laboratory longshore current experiment. The modified tracer advection diffusion equation should be considered for Eulerian ocean models to account properly for the surface wave effects.

Author Contributions: Conceptualization, S.Y. and Z.Z.; methodology, S.Y.; software, S.Y.; validation, S.Y. and Z.Z.; formal analysis, S.Y.; investigation, S.Y. and Z.Z.; resources, S.Y.; data curation, S.Y.; writing—original draft preparation, S.Y. and Z.Z.; writing—review and editing, S.Y. and Z.Y.; visualization, S.Y.; supervision, Z.Z. and Z.Y.; project administration, Z.Z.; funding acquisition, Z.Z. and Z.Y. All authors have read and agreed to the published version of the manuscript.

Funding: This research was funded by NSFC-Shandong Major Research Grant (Ref U1806227), the National Natural Science Foundation of China (Ref 51221961, 52101299) and Open Fund of State Key Laboratory of Coastal and Offshore Engineering (Grant No. LP2007).

Institutional Review Board Statement: Not applicable.

Informed Consent Statement: Not applicable.

Data Availability Statement: The data presented in this study are available on request from the corresponding author.

Conflicts of Interest: The authors declare no conflict of interest. The funders had no role in the design of the study; in the collection, analyses, or interpretation of data; in the writing of the manuscript, or in the decision to publish the results.

\section{References}

1. Stokes, G.G. On the theory of oscillatory waves. Trans. Camb. Phil. Soc. 1847, 8, 441-455.

2. Longuet-Higgins, M.S. Mass Transport in Water Waves. Philos. Trans. R. Soc. A-Math. 1953, 245, 535-581.

3. Monismith, S.G. A note on the potential transport of scalars and organisms by surface waves. Limnol. Oceanogr. 2004, 49, 1214-1217. [CrossRef]

4. Röhrs, J.; Christensen, K.H.; Vikebø, F.; Sundby, S.; Saetra, Ø.; Broström, G. Wave-induced transport and vertical mixing of pelagic eggs and larvae. Limnol. Oceanogr. 2014, 59, 1213-1227. [CrossRef]

5. $\quad$ Reniers, A.J.H.M.; MacMahan, J.H.; Thornton, E.B.; Stanton, T.P.; Henriquez, M.; Brown, J.W.; Brown, J.A.; Gallagher, E. Surf zone surface retention on a rip-channeled beach. J. Geophys. Res. Oceans 2009, 114, 1-12. [CrossRef]

6. Henderson, S.M.; Allen, J.S. Nearshore sandbar migration predicted by an eddy-diffusive boundary layer model. J. Geophys. Res. Oceans 2004, 109, 1-15. [CrossRef]

7. Hally-Rosendahl, K.; Feddersen, F.; Guza, R.T. Cross-shore tracer exchange between the surfzone and inner-shelf. J. Geophys. Res. Oceans 2014, 119, 4367-4388. [CrossRef]

8. Hally-Rosendahl, K.; Feddersen, F. Modeling surfzone to inner-shelf tracer exchange. J. Geophys. Res. Oceans 2016, 121, 4007-4025. [CrossRef] 
9. McWilliams, J.C.; Restrepo, J.M.; Lane, E.M. An asymptotic theory for the interaction of waves and currents in coastal waters. J. Fluid Mech. 2004, 511, 135-178. [CrossRef]

10. Taylor, G. Dispersion of soluble matter in solvent flowing slowly through a tube. Proc. R. Soc. Lond. Ser. A Math. Phys. Sci. 1953, $219,186-203$.

11. Law, A.W.K. Taylor dispersion of contaminants due to surface waves. J. Hydraul. Res. 2000, 38, 41-48. [CrossRef]

12. Van den Broeck, C. Taylor dispersion revisited. Phys. A Stat. Mech. Its Appl. 1990, 168, 677-696. [CrossRef]

13. Shen, L.D.; Zou, Z.L. A theoretical solution to dispersion coefficients in wave field. Ocean Eng. 2014, 88, 342-356. [CrossRef]

14. Winckler, P.; Liu, P.L.F.; Mei, C.C. Advective diffusion of contaminants in the surf zone. J. Waterw. Port Coast. Ocean Eng. 2013, 139, 437-454. [CrossRef]

15. Clark, D.B.; Feddersen, F.; Guza, R.T. Cross-shore surfzone tracer dispersion in an alongshore current. J. Geophys. Res. Oceans 2010, 115, C10035. [CrossRef]

16. Mei, C.C.; Fan, S.J.; Jin, K.R. Resuspension and transport of fine sediments by waves. J. Geophys. Res. Oceans 1997, $102,15807-15821$. [CrossRef]

17. Fischer, H.B.; List, J.E.; Koh, C.R.; Imberger, J.; Brooks, N.H. Mixing in Inland and Coastal Waters; Academic Press: San Diego, CA, USA, 1979; pp. 50-54.

18. Wood, I.R.; Bell, R.G.; Wilkinson, D.L. Ocean Disposal of Wastewater; World Scientific: Singapore, 1993 ; pp. 1-425.

19. Dean, R.G.; Dalrymple, R.A. Water Wave Mechanics for Engineers and Scientists; World Scientific Publishing Company: Singapore, 1991; pp. 295-305.

20. Chatwin, P.C. The approach to normality of the concentration distribution of a solute in a solvent flowing along a straight pipe. J. Fluid Mech. 1970, 43, 321-352. [CrossRef]

21. Dimou, K. Simulation of Estuary Mixing Using a Two-Dimensional Random Walk Model. Master's Thesis, Massachusetts Institute of Technology, Cambridge, MA, USA, 1989.

22. Hong, J.; Zhili, Z.; Dahong, Q.; Heiquan, S.; Shuping, W. The effects of wave-induced currents on the transport of pollutant outside and inside surf zone. Acta Oceanol. Sin. 2006, 28, 144-150. (In Chinese)

23. Lentz, S.J.; Fewings, M.; Howd, P.; Fredericks, J.; Hathaway, K. Observations and a model of undertow over the inner continental shelf. J. Phys. Oceanogr. 2008, 38, 2341-2357. [CrossRef]

24. Monismith, S.G.; Cowen, E.A.; Nepf, H.M.; Magnaudet, J.; Thais, L. Laboratory observations of mean flows under surface gravity waves. J. Fluid Mech. 2007, 573, 131-147. [CrossRef]

25. You, Z.J.; Wilkinson, D.L.; Nielsen, P. Near bed net drift under waves. In Proceedings of the 10th Australasian Conference on Coastal and Ocean Engineering, Auckland, New Zealand, 2-6 December 1991; pp. 183-186.

26. Castelle, B.; Michallet, H.; Marieu, V.; Leckler, F.; Dubardier, B.; Lambert, A.; Berni, C.; Bonneton, P.; Barthelemy, E.; Bouchette, F. Laboratory experiment on rip current circulations over a moveable bed: Drifter measurements. J. Geophys. Res. Oceans 2010, 115, 1-17. [CrossRef] 